\title{
Nuclear gene phylogeography using PHASE: dealing with unresolved genotypes, lost alleles, and systematic bias in parameter estimation
}

\author{
Ryan C Garrick*1,2, Paul Sunnucks ${ }^{3}$ and Rodney J Dyer ${ }^{1}$
}

\begin{abstract}
Background: A widely-used approach for screening nuclear DNA markers is to obtain sequence data and use bioinformatic algorithms to estimate which two alleles are present in heterozygous individuals. It is common practice to omit unresolved genotypes from downstream analyses, but the implications of this have not been investigated. We evaluated the haplotype reconstruction method implemented by PHASE in the context of phylogeographic applications. Empirical sequence datasets from five non-coding nuclear loci with gametic phase ascribed by molecular approaches were coupled with simulated datasets to investigate three key issues: (1) haplotype reconstruction error rates and the nature of inference errors, (2) dataset features and genotypic configurations that drive haplotype reconstruction uncertainty, and (3) impacts of omitting unresolved genotypes on levels of observed phylogenetic diversity and the accuracy of downstream phylogeographic analyses.
\end{abstract}

Results: We found that PHASE usually had very low false-positives (i.e., a low rate of confidently inferring haplotype pairs that were incorrect). The majority of genotypes that could not be resolved with high confidence included an allele occurring only once in a dataset, and genotypic configurations involving two low-frequency alleles were disproportionately represented in the pool of unresolved genotypes. The standard practice of omitting unresolved genotypes from downstream analyses can lead to considerable reductions in overall phylogenetic diversity that is skewed towards the loss of alleles with larger-than-average pairwise sequence divergences, and in turn, this causes systematic bias in estimates of important population genetic parameters.

Conclusions: A combination of experimental and computational approaches for resolving phase of segregating sites in phylogeographic applications is essential. We outline practical approaches to mitigating potential impacts of computational haplotype reconstruction on phylogeographic inferences. With targeted application of laboratory procedures that enable unambiguous phase determination via physical isolation of alleles from diploid PCR products, relatively little investment of time and effort is needed to overcome the observed biases.

\section{Background}

The increasing use of nuclear DNA (nDNA) sequences in phylogeographic studies, in combination with sequence data from a haploid organellar locus, has been driven by the considerable improvements in strength and accuracy of historical inference that multi-locus analyses can provide [1]. The development of conserved intron-spanning polymerase chain reaction ( $\mathrm{PCR}$ ) primers has facilitated amplification of low- or single-copy nuclear loci in non-

* Correspondence: ryan.garrick@yale.edu

1 Department of Biology, Virginia Commonwealth University, Richmond, Virginia 23284, USA

Full list of author information is available at the end of the article model species [2,3], and anonymous nuclear sequence loci have also been successfully applied in phylogeographic studies of diverse taxa (e.g., arthropods [4,5]; reptiles [6]; birds [7]). However, assaying nDNA sequence variation for reasonably large population-genetic sample sizes remains a major challenge [8]. There are several molecular laboratory techniques suitable for screening codominant markers by physically isolating sequencevariable alleles [9], but none have been broadly adopted owing to perceived or real logistical and/or technical limitations (e.g., high cost and time commitment, need for specialist equipment, difficulty resolving new or weakly 
amplifying alleles, and susceptibility to artefacts such as PCR recombination).

Algorithm-driven reconstruction of nuclear allele haplotypes following direct sequencing of diploid PCR products has become increasingly popular in phylogeographic and related applications (Additional file 1). These methods are based on the premise that the phase of alleles occurring in either homozygotes or heterozygotes that are polymorphic at only a single position can be resolved without ambiguity, and so this information can assist in resolving the phase of multi-site heterozygotes. One major advantage is that the per-locus cost of population screening is comparable to sequencing a fragment of animal mitochondrial DNA or plant chloroplast DNA, which is now quite routine and for which the necessary equipment and expertise are usually readily available. In addition, resolving power is thought to be quite good (all single nucleotide polymorphisms in a heterozygous genotype should be detectable). At present, one of the most widely used haplotype reconstruction methods is implemented in the software PHASE $[10,11]$. This Bayesian approach employs a neutral coalescent prior, making it suitable for population-genetic datasets, and it is able to accommodate recombination. Moreover, because PHASE uses Markov Chain Monte Carlo to sample the posterior distribution of potential haplotype pairs that could account for an observed ambiguous genotype, confidence probabilities for the phase of each segregating site and for each reconstructed haplotype pair can be estimated. In the context of genotype-phenotype association studies, PHASE has been shown to perform quite well with simulated and/or empirical human genetic datasets, but it is also frequently reported that rare haplotypes are susceptible to inference error [10-18]. This suggests that in applications where rare haplotypes are informative, computational approaches alone may be inadequate [19].

To date, few assessments of PHASE have been performed using non-coding gene regions, or datasets from species with complex evolutionary histories that are typical of non-human phylogeographic studies. However, patterns of variation at nuclear loci may be impacted by features of organismal biology such as effective population size, or past events and processes including population fragmentation, long-term isolation in refugia, and/or the existence of semipermeable landscape-level barriers to gene flow. For example, nuclear gene phylogeography of arthropods has revealed that extant taxa can exhibit highly polymorphic loci with many alleles segregating in large, deeply subdivided populations $[4,5,20,21]$, and hybridization at zones of secondary contact can potentially generate genotypes comprised of distantly-related or novel recombinant allele haplotypes [22]. Previously, Huang et al. [23] performed the first assessment of PHASE using a large population-genetic dataset from a non-model species (526 individuals of the migratory locust, Locusta migratoria). The anonymous single copy nuclear locus examined in that study was characterized by high overall heterozygosity $\left(H_{\mathrm{O}}=0.66\right)$ and many rare alleles, with $87.8 \%$ of the 115 distinct multi-site heterozygote genotypes present at frequencies $<1 \%$. The authors reported that $9 \%$ of individuals with ambiguous genotypes remained unresolved owing to confidence probability values below the chosen PHASE threshold of 0.95 . In a smaller scale study, Harrigan et al. [24] examined PHASE performance using a sample of 30 dabbling duck (Anas spp.) individuals with ambiguous genotypes, and found that $13-16.7 \%$ were unresolved when running the software with comparable settings.

Broadly speaking, genetic datasets known to be impacted by technical artefacts that operate in a non-random manner need to be analyzed and interpreted with caution (e.g., non-amplifying 'null' alleles [25]). However, an examination of papers focusing on phylogeography, hybridization and speciation indicates that in most empirical applications of PHASE, unresolved genotypes are simply omitted from the dataset (Table 1). Although this practice is generally considered to have negligible impacts on subsequent estimates of population genetic parameters and associated phylogeographic inferences [26], no formal assessment has been performed to date [27]. Indeed, there are reasons to believe that the duel loss of rare alleles and heterozygous genotypes could introduce systematic bias into downstream analyses. For example, consider the parameter $\Theta\left(4 N_{e} \mu\right.$ for diploid autosomal genes, where $N_{e}$ is effective population size and $\mu$ is the locus-specific per-generation mutation rate). This parameter is central to widely-used coalescent phylogeographic analyses (e.g., population size changes [28]; migration matrix estimation [29]; isolation-with-migration divergence modelling [30]), and $N_{e}$-values derived from $\Theta$ underpin simulation-based tests of alternative vicariance scenarios [4,21,31-33]. The estimation of $\Theta$ is heavily dependent on the number of segregating sites in a sample of sequences [34], and rare alleles usually contribute new segregating sites. It is therefore possible that computational haplotype reconstruction leads to the removal of a sufficiently large number of genotypes containing rare alleles so as to downwardly bias $\Theta$. The potential for systematic bias also extends to other analyses. For example, contrasts between within-population heterozygosity and the number of alleles form the basis of tests for recent genetic bottlenecks [35], and the frequency distributions of allele haplotypes [36] or pairwise nucleotide differences [37] are commonly used to distinguish exponential growth from population size constancy (Table 1). 
Table 1: Literature survey of empirical studies focusing on phylogeography, hybridization and speciation that used PHASE for haplotype reconstruction (see Additional file $\mathbf{4 4}$ for a complete list of references).

\begin{tabular}{|c|c|c|c|c|c|c|}
\hline & $\begin{array}{c}\text { Birds } \\
(N=15)\end{array}$ & $\begin{array}{l}\text { Herpetofauna } \\
\qquad(N=12)\end{array}$ & $\begin{array}{c}\text { Mammals } \\
(N=10)\end{array}$ & $\begin{array}{c}\text { Fish } \\
(N=8)\end{array}$ & $\begin{array}{c}\text { Invertebrates } \\
(N=15)\end{array}$ & $\begin{array}{l}\text { Total } \\
(N=60)\end{array}$ \\
\hline \multicolumn{7}{|c|}{ PHASE threshold used } \\
\hline 0.95 & 3 & - & 1 & 4 & 2 & 10 \\
\hline 0.90 & - & 4 & 1 & 1 & 2 & 8 \\
\hline $0.75-0.80$ & 4 & - & - & - & - & 4 \\
\hline 0.70 & 1 & 1 & - & - & - & 2 \\
\hline 0.60 & - & - & - & 2 & 3 & 5 \\
\hline $\begin{array}{l}\text { Best of replicate } \\
\text { runs }\end{array}$ & - & 1 & - & - & 2 & 3 \\
\hline Not reported & 7 & 6 & 8 & 1 & 6 & 28 \\
\hline \multicolumn{7}{|c|}{ Unresolved genotypes } \\
\hline $\begin{array}{l}\text { Excluded or coded } \\
\text { as missing }\end{array}$ & 5 & 5 & 1 & 5 & 3 & 19 \\
\hline $\begin{array}{l}\text { Resolved } \\
\text { experimentally }\end{array}$ & 2 & - & - & - & 2 & 4 \\
\hline $\begin{array}{l}\text { Included despite } \\
\text { uncertainty }\end{array}$ & 3 & - & - & - & - & 3 \\
\hline None present & 1 & 1 & - & 3 & 4 & 9 \\
\hline Not reported & 4 & 6 & 9 & - & 6 & 25 \\
\hline \multicolumn{7}{|c|}{ Experimental validation } \\
\hline Cloning & 1 & 2 & - & 1 & 4 & 8 \\
\hline Allele-specific PCR & 3 & - & - & - & - & 3 \\
\hline None & 11 & 10 & 10 & 7 & 11 & 49 \\
\hline \multicolumn{7}{|c|}{ Downstream analyses } \\
\hline Theta $(\Theta)^{b}$ & 14 & 6 & 9 & 4 & 7 & 40 \\
\hline $\begin{array}{l}\text { Nucleotide } \\
\text { diversity (П) }\end{array}$ & 9 & 7 & 9 & 4 & 7 & 36 \\
\hline $\begin{array}{l}\text { Neutrality or } \\
\text { population growth }\end{array}$ & 7 & 6 & 4 & 6 & 5 & 28 \\
\hline $\begin{array}{l}\text { Network or } \\
\text { phylogenetic tree }\end{array}$ & 10 & 9 & 9 & 6 & 10 & 44 \\
\hline
\end{tabular}

aUses all inferred haplotypes from the run with the best average goodness-of-fit

bIncludes studies that implicitly calculated theta as part of coalescent analyses (e.g., MIGRATE, FLUCTUATE, IM)

In the present paper we assess the performance of PHASE using five large nDNA sequence datasets from two Collembola species (Hexapoda), for which all genotypes have been resolved by laboratory procedures [38]. In addition, we analyze 35 simulated datasets with contrasting levels of polymorphism and, for the first time, examine the impact of unresolved genotypes and 'lost alleles' on downstream phylogeographic analyses. Out- comes are considered under PHASE confidence probability thresholds of 0.90 (i.e., the default value used by the software) and 0.60 , both of which are commonly used in the relevant empirical literature (Table 1). We conclude with a discussion of the complementarity of laboratorybased physical isolation of alleles and computational haplotype reconstruction. 


\section{Methods}

\section{Literature survey}

Papers citing Stephens et al. [10] or Stephens and Donnelly [11], and focusing on phylogeography, speciation or hybridization of natural populations of non-primate animals, were identified using Web of Science (accessed December 2009). Literature searches were conducted using the keywords "phylogeograph*", "speciation", or "gene flow" to find relevant papers in any journal, and by examining all citing articles in BMC Evolutionary Biology, Evolution, Molecular Ecology, and Molecular Phylogenetics and Evolution. If papers were within the scope of this survey, the Methods and Results sections were examined. Papers that primarily used PHASE in conjunction with non-coding nDNA sequence data were included, because we wanted to determine how the software was being used, and the types of downstream analyses that were performed using computationally-phased datasets.

\section{Datasets and polymorphism levels}

Our empirical nDNA sequence datasets were generated as part of a comparative phylogeographic investigation that included two saproxylic Collembola species $[5,39,40]$. Sequence variation at five nuclear loci was assayed in $>200$ individuals of either Acanthanura sp. nov. (three loci, prefix ' $U c^{\prime}$ ') or Pseudachorutinae gen. nov. sp. nov. (two loci, prefix 'Sm'; Table 2). These markers included an intron (elongation factor-1 $\alpha ; E F 1 \alpha$ ) and three non-coding anonymous loci. The number of nucleotides ranged from 92-266-bp, and alleles were phased by physically isolating them from diploid PCR products using single-stranded conformation polymorphism (SSCP) followed by targeted DNA sequencing [38,41]. This experimental approach minimizes artefacts that can arise from PCR recombination because it is cloning-free. Marker development and population screening methods are given in Garrick and Sunnucks [38]. Four of the five nuclear loci had alleles with several discontiguous insertion/deletion (indel) mutations. These were recoded using arbitrary nucleotide characters, with contiguous multi-base indels treated as a single event. In the present study, a 28-bp region of locus Sm2 (positions 138-165) was removed owing to unusually high polymorphism that exceeded the limits of PHASE. To reconstruct the sequences that would have been generated by direct sequencing of diploid PCR products from these five loci (i.e., with ambiguity codes), the two alleles from an individual genotype were collapsed into a consensus sequence using MESQUITE v2.5 [42]. Because this transformation of phase-known to ambiguous data includes no scoring error, our datasets represent idealized conditions.
Simulated DNA sequence datasets comprising 50 diploid genotypes sampled from a hypothetical panmictic population of constant size were generated with MESQUITE. Given that it is common for screening of $\mathrm{nDNA}$ loci to be performed on a subset of individuals taken from a larger phylogeographic study sample (e.g., 28 of the 60 studies included in our literature survey had total sample sizes of $\leq 100$ individuals per nDNA locus per species), our chosen sample size achieves a balance between statistical power and the reality of empirical datasets. Coalescent gene trees with 100 allele copies (i.e., terminal branches) were simulated backward-in-time within an isolated population of haploid $N_{e}=1,000$ individuals and age $=2,000$ organismal generations. This scheme of $2 N_{e}$ generations since isolation represents the theoretical expectation of the average time taken for alleles at a haploid locus to become reciprocally monophyletic on a gene tree. We did not use more complex models that included growth or decline because we wanted to assess PHASE performance under a best-case scenario, where the underlying assumptions of neutral coalescence within a single unstructured population of constant size were satisfied. Next, nucleotide characters (250-bp) were evolved forward-in-time along the branches of the coalescent gene trees using a HKY85 substitution model (root states and equilibrium base frequencies: A 0.30, C 0.20, G 0.15, $\mathrm{T} 0.35$; ts/tv $=2.5$ ). This substitution model and base frequency set is representative of our empirical Collembola data, and also typical of nDNA loci assayed for other organisms, as reported in the phylogeographic literature. To ensure independence, only one DNA sequence dataset was simulated per coalescent tree. During this process, scaling factors were used to convert branch lengths of coalescent gene trees (measured in organismal generations) into units that are typical of DNA sequence datasets (e.g., substitutions per site). To obtain levels of polymorphism that span the full range seen in empirical studies, scaling factors were determined by trial-anderror. We used the number of different alleles $\left(A_{\mathrm{N}}\right)$ and segregating sites $(S)$, calculated using DNASP v4.10.3 [43], as yardsticks of overall polymorphism. Scaling factor values between $1.0 \times 10^{-5}$ to $9.0 \times 10^{-6}$ generated a pool of 500 datasets, and from these we arbitrarily selected 35 datasets with $S$-values of $5,10,15,20,25,30$ or 35 (five datasets each; Table 2; Additional file 2). Within each of these 35 datasets, diploid genotypes were manually constructed by randomly pairing two haploid DNA sequences (i.e., alleles), to generate a diploid genotype. This represented random mating in a sexual outcrossing species. To mimic the phase-unknown genotypes produced from direct sequencing of diploid PCR products, consensus sequences for each genotype, with 
Table 2: Characteristics of five empirical and 35 simulated datasets used in the present study.

\begin{tabular}{|c|c|c|c|c|c|c|c|c|c|c|c|c|c|}
\hline \multirow{2}{*}{$\begin{array}{l}\text { Empirical } \\
\text { datasets }\end{array}$} & \multirow[b]{2}{*}{$N$} & \multirow{2}{*}{$\begin{array}{l}\text { Ambiguous } \\
\text { genotypes }\end{array}$} & \multicolumn{4}{|c|}{ Polymorphism measure } & \multirow{2}{*}{$\begin{array}{c}\text { Simulated } \\
\text { datasets }\end{array}$} & \multirow[b]{2}{*}{$N$} & \multirow{2}{*}{$\begin{array}{c}\text { Ambiguous } \\
\text { genotypes }\end{array}$} & \multicolumn{4}{|c|}{ Polymorphism measure } \\
\hline & & & $S$ & $A N$ & GN & HO & & & & $S$ & $A N$ & GN & HO \\
\hline \multicolumn{2}{|c|}{ Pseudachorutinae sp. } & & & & & & Sim01 & 50 & 5 & 5 & 6 & 6 & 0.26 \\
\hline Sm2 Pop1 & 80 & 0 & 2 & 3 & 4 & 0.03 & $\mathrm{Sim} 02$ & 50 & 14 & 5 & 6 & 11 & 0.58 \\
\hline Sm2 Pop2 & 61 & 0 & 2 & 3 & 3 & 0.02 & $\mathrm{Sim} 03$ & 50 & 2 & 5 & 6 & 9 & 0.66 \\
\hline Sm2 Pop3 & 118 & 2 & 9 & 5 & 7 & 0.14 & Sim04 & 50 & 9 & 5 & 6 & 9 & 0.50 \\
\hline Sm2 Pop4 & 62 & 0 & 2 & 4 & 4 & 0.03 & Sim05 & 50 & 6 & 5 & 6 & 8 & 0.50 \\
\hline Average & 80 & 1 & 4 & 4 & 5 & 0.06 & Sim06 & 50 & 25 & 10 & 8 & 12 & 0.76 \\
\hline SmEF1a Pop1 & 81 & 2 & 9 & 6 & 9 & 0.10 & Sim07 & 50 & 34 & 10 & 9 & 17 & 0.90 \\
\hline SmEF1a Pop2 & 60 & 8 & 6 & 3 & 4 & 0.13 & Sim08 & 50 & 25 & 10 & 10 & 22 & 0.76 \\
\hline SmEF1a Pop3 & 105 & 40 & 11 & 9 & 12 & 0.39 & Sim09 & 50 & 38 & 10 & 9 & 15 & 0.80 \\
\hline SmEF1a Pop4 & 54 & 9 & 6 & 6 & 8 & 0.22 & Sim10 & 50 & 31 & 10 & 9 & 23 & 0.80 \\
\hline Average & 75 & 15 & 8 & 6 & 8 & 0.21 & Sim11 & 50 & 36 & 15 & 11 & 17 & 0.82 \\
\hline \multicolumn{2}{|l|}{ Acanthanura sp. } & & & & & & $\operatorname{Sim} 12$ & 50 & 38 & 15 & 12 & 30 & 0.86 \\
\hline Uc3 Pop1 & 26 & 0 & 2 & 3 & 4 & 0.08 & $\operatorname{Sim} 13$ & 50 & 31 & 15 & 10 & 20 & 0.78 \\
\hline Uc3 Pop2 & 19 & 0 & 0 & 1 & 1 & 0.00 & $\operatorname{Sim} 14$ & 50 & 32 & 15 & 11 & 19 & 0.76 \\
\hline Uc3 Pop3 & 67 & 3 & 8 & 8 & 9 & 0.24 & $\operatorname{Sim} 15$ & 50 & 36 & 15 & 12 & 23 & 0.80 \\
\hline Uc3 Pop4 & 78 & 6 & 7 & 7 & 12 & 0.14 & $\operatorname{Sim} 16$ & 50 & 39 & 20 & 13 & 31 & 0.90 \\
\hline Uc3 Pop5 & 15 & 8 & 17 & 9 & 11 & 0.67 & $\operatorname{Sim} 17$ & 50 & 30 & 20 & 16 & 33 & 0.72 \\
\hline Average & 41 & 3 & 7 & 6 & 7 & 0.23 & $\operatorname{Sim} 18$ & 50 & 24 & 20 & 13 & 22 & 0.62 \\
\hline Uc180 Pop1 & 24 & 5 & 7 & 5 & 8 & 0.54 & Sim19 & 50 & 27 & 20 & 12 & 21 & 0.62 \\
\hline Uc180 Pop2 & 19 & 1 & 2 & 2 & 2 & 0.05 & $\operatorname{Sim} 20$ & 50 & 41 & 20 & 15 & 30 & 0.86 \\
\hline Uc180 Рop3 & 67 & 0 & 2 & 3 & 3 & 0.03 & $\operatorname{Sim} 21$ & 50 & 40 & 25 & 19 & 37 & 0.90 \\
\hline Uc180 Pop4 & 78 & 0 & 1 & 2 & 2 & 0.01 & $\operatorname{Sim} 22$ & 50 & 32 & 25 & 15 & 22 & 0.70 \\
\hline Uc180 Pop5 & 15 & 0 & 0 & 1 & 1 & 0.00 & $\operatorname{Sim} 23$ & 50 & 30 & 25 & 17 & 32 & 0.72 \\
\hline Average & 41 & 1 & 2 & 3 & 3 & 0.13 & $\operatorname{Sim} 24$ & 50 & 20 & 25 & 13 & 21 & 0.70 \\
\hline UcEF1a Pop1 & 26 & 6 & 6 & 6 & 8 & 0.23 & $\operatorname{Sim} 25$ & 50 & 35 & 25 & 18 & 29 & 0.84 \\
\hline UcEF1a Pop2 & 19 & 1 & 7 & 4 & 4 & 0.16 & $\operatorname{Sim} 26$ & 50 & 40 & 30 & 20 & 39 & 0.84 \\
\hline UcEF1a Рop3 & 67 & 21 & 15 & 10 & 11 & 0.36 & $\operatorname{Sim} 27$ & 50 & 41 & 30 & 17 & 36 & 0.90 \\
\hline UcEF1a Pop4 & 78 & 12 & 14 & 10 & 11 & 0.26 & $\operatorname{Sim} 28$ & 50 & 32 & 30 & 19 & 29 & 0.82 \\
\hline UcEF1a Pop5 & 15 & 0 & 0 & 1 & 1 & 0.00 & $\operatorname{Sim} 29$ & 50 & 40 & 30 & 21 & 38 & 0.90 \\
\hline Average & 41 & 8 & 8 & 6 & 7 & 0.20 & $\operatorname{Sim} 30$ & 50 & 41 & 30 & 22 & 42 & 0.92 \\
\hline $\operatorname{Sm} 2$ & 321 & 2 & 13 & 11 & 14 & 0.07 & $\operatorname{Sim} 31$ & 50 & 37 & 35 & 17 & 35 & 0.88 \\
\hline SmEF1a & 300 & 59 & 17 & 17 & 27 & 0.24 & $\operatorname{Sim} 32$ & 50 & 41 & 35 & 17 & 36 & 0.94 \\
\hline Uc3 & 205 & 17 & 26 & 17 & 30 & 0.19 & $\operatorname{Sim} 33$ & 50 & 41 & 35 & 16 & 32 & 0.88 \\
\hline Uc180 & 203 & 6 & 12 & 9 & 13 & 0.08 & Sim34 & 50 & 46 & 35 & 20 & 38 & 0.92 \\
\hline UCEF1a & 205 & 40 & 27 & 21 & 32 & 0.26 & $\operatorname{Sim} 35$ & 50 & 38 & 35 & 15 & 28 & 0.80 \\
\hline Average & 247 & 25 & 19 & 15 & 23 & 0.17 & Average & 50 & 31 & 20 & 13 & 25 & 0.77 \\
\hline
\end{tabular}

Genetically distinct populations of two Collembola species identified previously $[5,40]$ were pooled prior to reanalysis using PHASE, but are separated here for comparison with the single-population simulated datasets. $N$ is the number of diploid individuals, and 'ambiguous genotypes' are those containing at least two heterozygous sites. Polymorphism measures are: $S$, number of segregating sites; $A_{N}$, number of different alleles; $G_{N}$, number of different genotypes; $H_{\mathrm{O}}$, observed heterozygosity. 
standard IUPAC nucleotide ambiguity codes at heterozygous positions, were generated in MESQUITE (as for the empirical Collembola data, above). In addition to calculating $S$ and $A_{\mathrm{N}}$ for each empirical and simulated dataset, we also quantified overall polymorphism via the number of different genotypes $\left(G_{\mathrm{N}}\right)$, and observed heterozygosity $\left(H_{\mathrm{O}}\right)$. Although the four summary statistics are non-independent (Additional file 3), they do reflect different components of the standing genetic variation, and so it is useful to investigate their relationships with PHASE performance.

\section{PHASE error, unresolved genotypes and lost alleles}

Simulated and empirical datasets were analyzed using PHASE v2.1.1 [10,11], with files formatted in SeqPHASE [44]. We employed the MR model which makes explicit allowance for intragenic recombination, and compared to the non-recombination model, it performs better. However, the primary reason for choosing this model was because assessing evidence for recombinant alleles (c.f. making a priori assumptions about their absence) is important when using nDNA sequences in empirical population-level studies. For tri-allelic single nucleotide polymorphisms, the parent-independent mutation model was used. Runs consisted of 500 iterations as burn-in, 500 main iterations, and thinning interval $=1$. Datasets were run three times with a different starting seed, and consistency across runs was checked by eye. The replicate with the best average goodness-of-fit value was used in subsequent analyses.

In the present paper we focus on the accuracy of PHASE in reconstructing whole haplotypes (c.f. individual single nucleotide polymorphisms) and diploid genotypes under two alternative thresholds, 0.60 and 0.90 . These values encompass commonly used cut-offs (Table 1 ; note that the PHASE default is 0.90 , and if not explicitly reported by studies included in the literature survey, we assumed the default value was used). Under each threshold, three aspects of performance were investigated:

$N_{\text {ERR }}$ Number of individuals with a confidently resolved (above-threshold) haplotype pair that included errors.

$N_{\text {LCP }}$ Number of individuals with genotypes that remained unresolved due to low confidence probability values (i.e., below-threshold). We also quantified the number of these individuals with correctly and incorrectly inferred haplotype pairs $\left(N_{\mathrm{LCP}}\right.$ correct and $N_{\text {LCP incorrect }}$ respectively).

$N_{\text {LOST }}$ Number distinct gene lineages (i.e., different alleles) that were present in the original phase-known dataset but were lost as a consequence of excluding individuals with unresolved $\left(N_{\mathrm{LCP}}\right)$ genotypes.

\section{Error}

There have been contrasting reports regarding the relationship between PHASE error and the number of heterozygous sites in an ambiguous genotype (i.e., negative [14]; positive [18]; no relationship [23]), so we assessed the correlation between the latter and the sum of $N_{\text {ERR }}+N_{\text {LCP incorrect. }}$. We also examined which type of mistake was most prevalent in cases where PHASE haplotype pair error is analogous to false positives $\left(N_{\mathrm{ERR}}\right)$, as well for those where the software appropriately indicated low confidence probability values $\left(N_{\mathrm{LCP}}\right.$ incorrect $)$, using the following four categories that capture all observed mistakes:

$E_{\mathrm{COR}+\text { NOV }}$ One haplotype is correct, the other is novel (did not previously exist).

$E_{\mathrm{COR}+\text { MIS }}$ One haplotype is correct, the other is misidentified (previously existing).

$E_{\mathrm{MIS}+\text { MIS }}$ Both haplotypes are misidentified.

$E_{\mathrm{MIS}+\text { NOV }}$ One haplotype is misidentified, the other is novel.

\section{Unresolved genotypes}

Intuitively, phase determination for highly variable nuclear sequence loci should be more challenging than for relatively invariable markers. Despite this expectation, there is still a relatively limited understanding of the specific features of a dataset that drive uncertainty associated with haplotype pair reconstruction (i.e., which particular aspects of genetic variation), and so this warrants further investigation. Similarly, although the presence of rare alleles is known to contribute to difficulties with inferring phase of segregating sites in multi-site heterozygotes, there is little information on the importance of the specific genotypic configurations in which rare alleles occur. First, to determine whether relationships between $N_{\mathrm{LCP}}$ and each of the four measures of dataset polymorphism levels $\left(S, A_{\mathrm{N}}, G_{\mathrm{N}}\right.$ and $\left.H_{\mathrm{O}}\right)$ exhibit different relative strengths, regression analyses were performed, with comparisons made using $R^{2}$-values. Second, for each unresolved genotype in our simulated datasets, population frequencies of the two constituent alleles were calculated. These data were then summarized in box plots to assess the prevalence of small versus large asymmetries (e.g., pairing of two rare alleles, c.f. pairing of a common and a rare allele). Corresponding plots were constructed for empirical datasets.

\section{Lost alleles}

We employed two analytical approaches to assess the impact of omitting unresolved $\left(N_{\mathrm{LCP}}\right)$ genotypes on 'phylogenetic diversity' [45]. To examine overall loss of distinct gene lineages, $N_{\text {LOST }}$ values were scaled by the number of different alleles in each original dataset, and a regression analysis was performed using corresponding $N_{\mathrm{LCP}}$ values as the predictor variable. We also investigated 
whether lost alleles tended to be more divergent than other alleles in the dataset, as measured by proportion of nucleotide differences between a pair of sequences (uncorrected $p$ ) calculated in MEGA v4.0 [46]. We chose this simple measure of genetic distance (c.f. HKY-corrected distances) because the vast majority of polymorphic sites (97\%) were consistent with an infinite-alleles mutation model, and uncorrected $p$ is often used for nDNA sequence datasets in population-level studies. We plotted the difference between the mean from only those pair-wise comparisons involving the lost allele under consideration $\left(p_{\text {LOST }}\right)$ and the mean of all pair-wise comparisons within a dataset $\left(p_{\text {DATASET }}\right)$. Datasets with $>1$ lost allele have non-independent data points because multiple comparisons are made using the same $p_{\text {DATASET }}$ value. In these cases (24 simulated and one empirical dataset), $p_{\text {LOST }}$ values were first summed, and then deducted from $p_{\text {DATASET }}$, thereby generating a single data point per dataset.

\section{Standard phylogeographic analyses}

The omission of unresolved genotypes from a genetic dataset could potentially introduce biases into downstream phylogeographic analyses. We assessed the magnitude and directionality of differences in point estimates of two measures of population genetic diversity (Watterson's [34] $\Theta_{W}$, and Nei's [47] $\pi$ ), as well as two measures of demographic growth or neutrality (Tajima's [48]D and Fu's $\left.[36] F_{S}\right)$. Empirical studies focusing on phylogeography, hybridization and speciation that use PHASE often estimate these parameters (Table 1). For each simulated dataset, parameter values were calculated in DNASP for the original phase-known dataset, and then recalculated after removing unresolved genotypes under each of the two thresholds. Significance of differences in parameter values was assessed via one-tailed paired t-tests, implemented in STATSDIRECT v2.7.7 http://www.statsdirect.com. Finally, we investigated the extent to which lost alleles can alter estimated root probabilities in intraspecific haplotype networks. To do this, statistical parsimony networks constructed for simulated datasets using TCS v1.21 [49] with the 95\% confidence criterion enforced. The allele with the highest outgroup weight was determined for each of the original phase-known datasets, and then compared to results obtained when constructing statistical parsimony networks for the corresponding 'pruned' (90- $N_{\mathrm{LCP}}$ and $\left.60-N_{\mathrm{LCP}}\right)$ datasets.

\section{Results}

\section{Literature survey}

Sixty papers from 18 journals met our search criteria (Table 1 and Additional file 4). Each major vertebrate group and a diversity of invertebrates were represented.
Based on those studies that reported how unresolved genotypes were treated, the most common course of action is complete exclusion. In some cases, the overall reduction in dataset size per locus was considerable (e.g., $21.9 \%$ [50], $13.6 \%$ [51], up to $8.5 \%$ [52]). Few studies used laboratory procedures in conjunction with computational phasing. Although the 60 papers used a variety of population-level analyses, $\Theta$ and $\pi$ were frequently estimated. Many studies also examined evidence for selection acting on nDNA and/or demographic growth using Fu's $F_{\mathrm{S}}$, Tajima's $D$, mismatch distributions or related statistical procedures, and phylogenetic relationships among alleles were often represented as networks or bifurcating trees.

\section{Datasets and polymorphism levels}

Simulated datasets encompassed a broad spectrum of polymorphism levels $\left(A_{\mathrm{N}}=6-22, G_{\mathrm{N}}=6-42, H_{\mathrm{O}}=0.26\right.$ $0.94)$, and the number of ambiguous genotypes ranged from 2-46 (Table 2). These polymorphism levels were considerably higher than those for each of the genetically-distinct Collembola populations, but at least superficially similar to the mean values obtained for the Collembola data pooled across loci and species (i.e., $S=$ $19, A_{\mathrm{N}}=15$, and $G_{\mathrm{N}}=23$; Table 2). Most papers included in the literature survey simultaneously analyzed multipopulation datasets with PHASE, and reported $A_{\mathrm{N}}$ values were usually within or slightly above the range seen in our empirical datasets. In this context, the present paper should provide a useful framework for understanding impacts of unresolved genotypes and lost alleles on downstream phylogeographic analyses. On the other hand, large differences exist between empirical versus simulated values of $H_{\mathrm{O}}$, and we recognize that this discrepancy warrants some caution when drawing generalizations from present study. A likely reason for this discrepancy is the presence of geographic substructure within and among Collembola populations. Generally speaking, where geographic substructure exists, rare alleles can occur at locally high frequencies with at least some in homozygous form, rather than at uniformly low frequencies and always as heterozygotes. Ultimately this would reduce heterozygosity, and in particular, may result in fewer ambiguous genotypes that contain rare alleles never before seen in homozygous form.

\section{PHASE error, unresolved genotypes and lost alleles}

PHASE inferences were consistent across replicate runs, indicating that search settings were adequate. Where several alternative solutions for a particular haplotype pair were recovered in the replicate with the best average goodness-of-fit, we used the reconstruction with the highest confidence probability value. If necessary, we randomly selected one of the equally well-support alternatives. 


\section{Error}

The relationship between PHASE error $\left(N_{\text {ERR }}+N_{\text {LCP incor- }}\right.$ rect) and the number of heterozygous sites in an ambiguous genotype was negative for simulated and empirical datasets $(r=-0.692$ and $r=-0.655$, respectively; Figure 1 solid circles vs. open circles). Under the 0.90 threshold, false positives $\left(N_{\text {ERR }}\right)$ were very rare when considering all simulated datasets together (5/1077 ambiguous genotypes $=0.5 \%)$, and use of the lower stringency threshold had little impact $(9 / 1077=0.8 \%$; Table 3$)$. Similarly, no increase in false positives was seen for the pooled empirical data $(3 / 126=2.4 \%$ error under both thresholds). For those datasets in which false positives occurred, error rates were as high as $12.5 \%$ (Uc3), but all others were $<10 \%$ irrespective of PHASE threshold (Sim06, up to 8\%; Sim11, 8.3\%; Sim33, 4.9\%; UcEF-1 $\alpha, 2.4 \%)$. Although only $2-3$ of 35 simulated datasets (5.7-8.6\%) included at least one false positive, this proportion was higher for the empirical datasets $(2 / 5=40 \%$ under both thresholds). In all cases, the most prevalent type of mistake was where one haplotype is misidentified and the other is novel

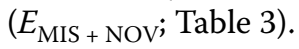

\section{Unresolved genotypes}

Most simulated datasets had some genotypes that were not resolved at or above the specified confidence levels ( $N=33$ and 30 datasets under thresholds 0.90 and 0.60 , respectively). Under the 0.90 threshold, the number of these unresolved genotypes $\left(90-N_{\mathrm{LCP}}\right)$ per simulated dataset ranged from $0-12$ (mean $=3.37)$. Overall, $62.7 \%$ of these unresolved genotypes were nonetheless inferred correctly. Lowering the PHASE threshold to 0.60 led to reductions in the number of unresolved genotypes, but also in the proportion of correctly reconstructed haplotype pairs $\left(60-N_{\mathrm{LCP}}\right.$ range: $0-7$, mean: 2.34 , proportion correct: $51.2 \%)$. The same general trends were seen in the empirical datasets $\left(90-N_{\mathrm{LCP}}\right.$ range: $0-7$, mean: 3 , proportion correct: $80 \% ; 60-N_{\mathrm{LCP}}$ range: $0-3$, mean: 0.80 , proportion correct: $25 \%)$. When $N_{\mathrm{LCP}}$ was represented as a proportion of the number of ambiguous genotypes (i.e., multi-site heterozygotes) present in each simulated dataset, the frequency distribution for the percentage of ambiguous genotypes that were not resolved under the 0.90 PHASE threshold (mode $=10-14 \%$ category; Figure 2 , pale grey bars) is slightly off-set to the right compared to that of the 0.60 threshold (mode $=5-9 \%$ category; Figure 2, dark grey bars). This indicates that enforcement of a higher-stringency limit on acceptable confidence probability scores generally leads to an increased proportion of unresolved genotypes per dataset.

For the simulated data, regression analyses showed that significant positive relationships exist between each of the four polymorphism measures and $N_{\text {LCP }}$ (Additional file 5). With the exception of $H_{\mathrm{O}}$ (Additional file 5, G-H), the overall strength of each relationship increased considerably as the PHASE threshold was decreased from 0.90 to 0.60 , indicating that inclusion of 'marginal' haplotype pairs in the $N_{\text {LCP }}$ category (i.e., those with confidence probability scores of 0.61-0.89) mostly contributes noise. For both thresholds, the strongest predictor variable was $A_{\mathrm{N}}$ (Additional file 5, C-D; 90- $N_{\mathrm{LCP}}$ : slope $=0.264$, d.f. $=$ $1,33, F=11.0, P=0.002, R^{2}=0.25 ; 60-N_{\mathrm{LCP}}$ : slope $=0.246$, d.f. $\left.=1,33, F=24.7, P<0.001, R^{2}=0.43\right)$. Regressions of the empirical data showed no significant relationships between $N_{\mathrm{LCP}}$ and any of the four polymorphism measures, but these analyses were limited by small sample sizes ( $N=5$ data points). Qualitatively, only one predictor variable $\left(H_{\mathrm{O}}\right)$ showed a marked discrepancy between slopes of simulated versus empirical datasets (slope = 7.30 vs. 24.78), but this was limited to the higher PHASE threshold, whereas there was essentially no difference under the lower stringency settings (slope $=5.62$ vs. 5.97 ; Additional file 5). Overall, the simulated datasets generated under panmixia, and the empirical datasets that include considerable substructuring, show similar relationships with polymorphism measures.

The majority of unresolved genotypes in simulated datasets included at least one singleton allele (70\% of 90 $N_{\text {LCP }}$ and $94 \%$ of $\left.60-N_{\text {LCP }}\right)$. A considerable number of these $\left(19.5 \%\right.$ of $90-N_{\mathrm{LCP}}$ and $20.7 \%$ of $\left.60-N_{\mathrm{LCP}}\right)$ included a second 'rare' allele (i.e., frequency $<0.05$ ), indicating that genotypic configurations involving two low-frequency alleles are disproportionately represented (Figure 3). Other aspects of $N_{\text {LCP }}$ genotypic configurations also indicate that they are non-random with respect to population allele frequencies. For example, there is usually no overlap between the inner 50\% quantile calculated for 'frequency of most common allele in an unresolved genotype' versus that calculated for 'frequency of most common allele in the dataset' (Figure 3 left panel). The two exceptions (i.e., rarest allele frequency $=0.02$ and 0.06 under PHASE thresholds 0.90 and 0.60 , respectively; Figure 3) both have upper and lower 25\% quantiles that do not extend beyond their inner $50 \%$ quantile, and so are likely to have been impacted by small sample sizes. If these two tentatively unreliable box plots are ignored, only two meaningful comparisons across PHASE thresholds are possible for the simulated datasets (i.e., 'frequency of rarest allele' categories 0.01 and 0.02). Qualitatively, empirical datasets show the same general patterns relating to genotypic configuration of unresolved genotypes seen in simulated data, and PHASE thresholds do not appear to alter outcomes (Figure 3 right panel).

Lost alleles

The number of lost alleles per simulated dataset ranged from $1-10$ or $1-7$ ( 0.90 or 0.60 threshold, respectively; 
Table 3: Accuracy of PHASE haplotype pair reconstructions.

\begin{tabular}{|c|c|c|c|c|c|}
\hline \multirow[b]{2}{*}{ Dataset } & \multirow{2}{*}{$\begin{array}{l}\text { Error } \\
\text { category }\end{array}$} & \multicolumn{2}{|c|}{0.90 threshold } & \multicolumn{2}{|c|}{0.60 threshold } \\
\hline & & 90-NERR & $\begin{array}{l}\text { 90-NLCP } \\
\text { incorrect }\end{array}$ & 60-NERR & $\begin{array}{l}\text { 60-NLCP } \\
\text { incorrect }\end{array}$ \\
\hline \multirow[t]{5}{*}{ Simulated } & $E_{\mathrm{COR}+\mathrm{NOV}}$ & - & 1 & - & 1 \\
\hline & $E_{\mathrm{COR}+\mathrm{MIS}}$ & 2 & - & 2 & - \\
\hline & $E_{\mathrm{MIS}+\mathrm{MIS}}$ & - & 2 & - & 2 \\
\hline & $E_{\mathrm{MIS}+\mathrm{NOV}}$ & 3 & 41 & 7 & 37 \\
\hline & Total & 5 & 44 & 9 & 40 \\
\hline \multirow[t]{5}{*}{ Empirical } & $E_{\mathrm{COR}+\mathrm{NOV}}$ & - & - & - & - \\
\hline & $E_{\mathrm{COR}+\mathrm{MIS}}$ & - & - & - & - \\
\hline & $E_{\mathrm{MIS}+\mathrm{MIS}}$ & - & - & - & - \\
\hline & $E_{\mathrm{MIS}+\mathrm{NOV}}$ & 3 & 3 & 3 & 3 \\
\hline & Total & 3 & 3 & 3 & 3 \\
\hline
\end{tabular}

False positives $\left(N_{\mathrm{ERR}}\right)$ and incorrect reconstructions that had appropriately low confidence probabilities $\left(N_{\mathrm{LCP}}\right.$ incorrect $)$ are reported for simulated and empirical datasets under two alternative PHASE confidence probability thresholds. Error categories reflect the nature of inference mistakes, and are described in Methods.

mean $=3$ for both). Regression analyses showed a significant positive relationship between $N_{\mathrm{LCP}}$ and reductions in the number of gene lineages in simulated datasets (scaled $N_{\text {LOST }}$; Figure 4 solid circles). The nature of this relationship was similar for both PHASE thresholds $\left(90-N_{\mathrm{LCP}}\right.$ : slope $=0.041$, d.f. $=1,33, F=92.2, R^{2}=0.74, P<<0.0001$, Figure 4 top; $60-N_{\mathrm{LCP}}$ : slope $=0.048$, d.f. $=1,33, F=92.9$, $R^{2}=0.74, P<0.0001$, Figure 4 bottom), indicating that

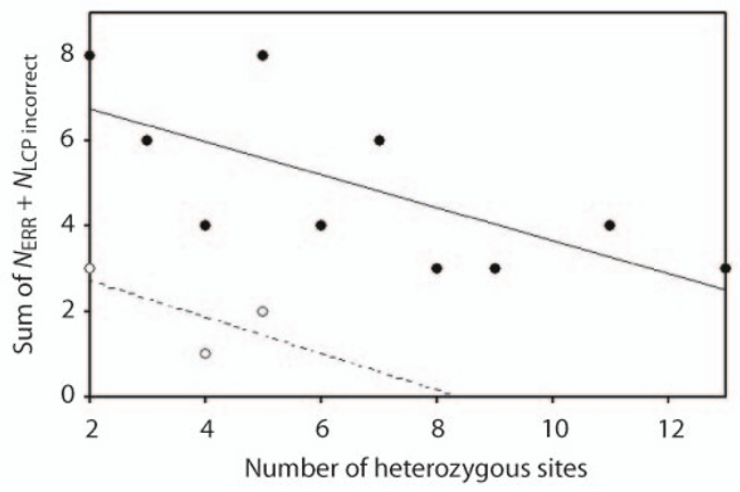

Figure 1 Relationship between the number of heterozygous sites in an ambiguous genotype ( $x$-axis) and haplotype pair reconstruction error ( $\boldsymbol{y}$-axis). Simulated datasets (solid circles) and empirical datasets (open circles) both showed strong negative correlations ( $r$ $=-0.692$ and $r=-0.655$, respectively). The plot is identical for the 0.90 and 0.60 PHASE confidence probability thresholds (the latter not shown). inclusion of 'marginal' haplotype pairs in the $90-N_{\mathrm{LCP}}$ category contributes to this trend. This systematic loss of phylogenetic diversity with increasing $N_{\text {LCP }}$ was mirrored by the empirical data under the 0.60 threshold, but unexpectedly, not the 0.90 threshold (Figure 4 open circles).

When considering pairwise sequence divergences among alleles, there are indications that lost alleles tend to be more divergent than retained alleles in simulated datasets. Although distributions of the difference in mean $p$-distance $\left(p_{\text {LOST }}-p_{\text {DATASET }}\right)$ under both PHASE thresh-

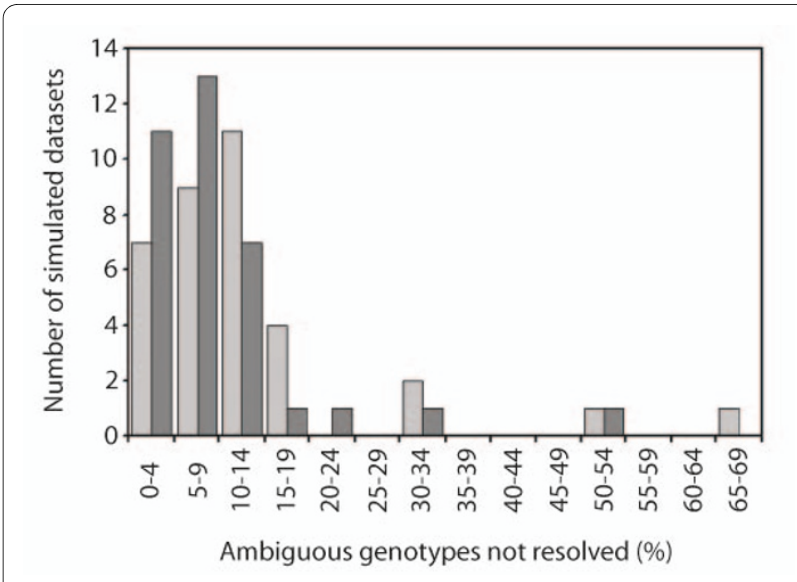

Figure 2 Frequency distribution of the number of unresolved genotypes $\left(N_{\mathrm{LCP}}\right)$ represented as a proportion of the total number of ambiguous genotypes present in each simulated dataset. Distributions for PHASE confidence probability thresholds 0.90 and 0.60 are shown in pale grey and dark grey, respectively. 
olds show modal values centered on zero (histogram category -0.002 to +0.002$)$, the plots are right-skewed (Figure 5). This trend was also seen when summarizing the data using other measures of central tendency and degree of asymmetry $\left(0.90\right.$ threshold: $p_{\text {LOST }}-p_{\text {DATASET }}$ mean $=0.0038$, skew $=0.520 ; 0.60$ threshold: mean $=$ 0.0044 , skew $=0.466$ ). Loss of divergent alleles owing to below-threshold PHASE confidence probability scores of reconstructed haplotype pairs also extends to empirical datasets $\left(U c 180, N_{\mathrm{LOST}}=1, U c E F-1 \alpha, N_{\mathrm{LOST}}=3 ; p_{\mathrm{LOST}}-\right.$ $p_{\text {DATASET }}$ mean $=0.0060$ under both thresholds).

\section{Standard phylogeographic analyses}

Estimated values of four commonly-used population genetic parameters showed marked directional biases as a consequence of omitting unresolved genotypes from simulated datasets, and the magnitude of these biases was similar for the two PHASE thresholds (Figure 6). The two measures of genetic diversity $\left(\Theta_{\mathrm{W}}\right.$ and $\left.\pi\right)$ were increasingly underestimated as more unresolved genotypes were omitted, whereas the two measures of demographic growth or neutrality (Tajima's $D$ and Fu's $F_{\mathrm{S}}$ ) were progressively overestimated. Regression analyses confirmed that relationships were significant (all $P$-values $<0.001$ ), with the strongest relationships seen for decreases in $\Theta_{\mathrm{W}}$ $\left(R^{2}=0.64\right.$ and 0.70 , Figure $6 \mathrm{~A}-\mathrm{B}$, respectively) and increases in Fu's $F_{\mathrm{S}}\left(R^{2}=0.78\right.$ and 0.83 , Figure $6 \mathrm{G}-\mathrm{H}$, respectively). Moreover, paired t-tests showed that parameter values obtained after removing unresolved genotypes differed significantly from those of the corresponding phase-known datasets (all $P$-values $<0.0001$ with d.f. $=32$; Figure 6A: $\mathrm{t}=6.39$, Figure 6B: $\mathrm{t}=6.67$, Figure $6 \mathrm{C}: \mathrm{t}=4.71$, Figure 6D: $\mathrm{t}=4.96$, Figure $6 \mathrm{E}: \mathrm{t}=-7.47$, Figure 6F: $\mathrm{t}=-8.03$, Figure 6G: $\mathrm{t}=-8.07$, Figure $6 \mathrm{H}: \mathrm{t}=-$ 7.62). When considering all simulated datasets together, the maximum downward bias affecting $\Theta_{\mathrm{W}}$ and $\pi$ were relatively small considering the 'true' mean values estimated from the original phase-known datasets (mean $\Theta_{\mathrm{W}}$ $=0.0160$, bias $=0.0086 ;$ mean $\pi=0.0157$, bias $=0.0024)$. However, at the level of individual datasets, reductions in $\Theta_{\mathrm{W}}$ were $\geq 20 \%$ of the 'true' value for $9-10$ of the 33 simulated datasets with unresolved genotypes. For the pooled simulated data, the two demographic growth or neutrality parameters showed large maximum upward biases compared to the true values (mean $D=-0.057$, bias = 1.135; mean $F_{\mathrm{S}}=-0.412$, bias $=4.847$ ).

In our study, the omission of unresolved genotypes led to changes in the rooting of haplotype networks in three of 35 simulated datasets (8.6\%). The example of a root switching error shown in Figure 7 was also seen in a dataset ('Sim 26') that produced a more complex set of three disconnected networks. In the latter case, switch errors affected two of these networks. However, changes in the rooting of haplotype networks do not always involve root switching. For example, one of our simulated datasets ('Sim33') produced two disconnected networks, but following removal of unresolved genotypes, these were subsequently split into four (the same outcome resulted under both $90-N_{\mathrm{LCP}}$ and $60-N_{\mathrm{LCP}}$ thresholds). This was caused by the loss of a single allele that occupied an important position in one of the original networks-a position that served as a link between otherwise distantly-related alleles (not shown).

\section{Discussion}

\section{Haplotype reconstruction errors}

False positive PHASE inferences, defined here as ambiguous genotypes for which above-threshold haplotype pair reconstructions included errors, were generally very low (Table 3). At the level of individual datasets, rates were usually $<10 \%$ (with the exception of one empirical dataset, $U_{c} 3$ ). Considering all simulated datasets together, false positive rates were $<1 \%$, and $<3 \%$ for the pooled empirical data. Haplotype pair reconstruction errors usually involved misidentifying an existing allele coupled with the creation of a novel allele, such that both inferred haplotypes were incorrect (Table 3). The misidentified allele was usually inferred to be the most common allele in the dataset (35-39\% or $83 \%$ of $E_{\mathrm{MIS}}+\mathrm{NOV}$ for simulated or empirical datasets, respectively). Our data also showed a negative correlation between the number of heterozygous sites and PHASE error (c.f. [18] and [23]), and so even two-site heterozygotes can be difficult to reconstruct accurately (Figure 1). However, given the low false positive rates, overestimation of common allele frequencies is unlikely to impact downstream analyses.

Low false positives from PHASE have been reported in several studies based on simulated data and/or well-characterized functional loci in humans [10-18]. The present work, together with two recent papers [23,24], extends these assessments to non-coding anonymous or intronic nDNA from other organisms. The consistently good performance suggests that PHASE is robust to some violations of the underlying neutral coalescent model (e.g., selection, kin clustering, population structure [53]).

\section{Drivers of uncertainty}

Although highly polymorphic nuclear sequence loci often carry considerable phylogeographic signal, they also tend to produce challenging datasets for computational haplotype reconstruction. The number of different alleles $\left(A_{\mathrm{N}}\right)$ is a particularly strong predictor of the number of unresolved $\left(N_{\mathrm{LCP}}\right)$ genotypes because allele-rich datasets usually contain many rare alleles. Indeed, the presence of a rare allele in an ambiguous genotype is perhaps the single most important determinant of PHASE's ability to confidently and accurately reconstruct haplotype pairs [19]. In 

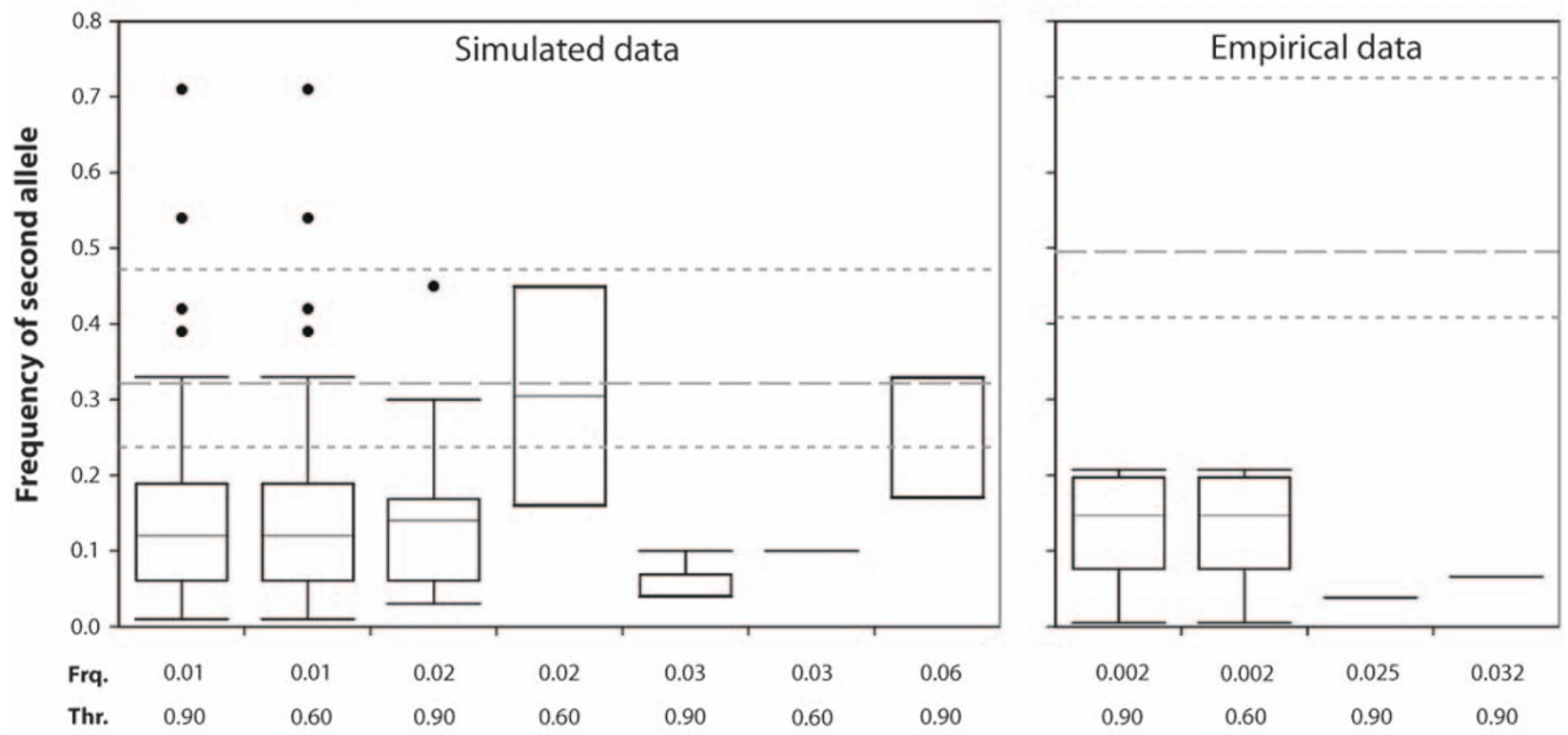

Frequency of rarest allele in low confidence probability genotype (Frq.), and PHASE threshold (Thr.)

Figure 3 Genotypic configurations of unresolved genotypes in simulated and empirical datasets. Population allele frequency values for the more common allele in an unresolved genotype are shown on a continuous scale ( $y$-axis), with a separate box plot drawn for each observed value of the rarer allele in an unresolved genotype ( $x$-axis). In each plot, the box represents the inner $50 \%$ quantile (median marked by a solid black line), and the whiskers represent the upper and lower $25 \%$ quantile, excluding outliers (solid black circles). For comparative purposes, the population frequency of the most common allele present in each dataset was used to calculate an overall median and inner $50 \%$ quantile (dashed grey lines) for simulated and empirical datasets.

populations that have undergone relatively recent and rapid range expansion, coalescent theory predicts an excess of low-frequency haplotypes [28,36,37], and so demographic history may contribute to the number of unresolved genotypes.

Our investigation of the influence of rare alleles on PHASE confidence probabilities indicated that genotypic configurations involving two low-frequency alleles were disproportionately represented in the pool of unresolved genotypes (Figure 3). In out-crossing panmictic populations, these heterozygotes will tend to be very uncommon. However, in hybrid zones, the propensity for rare alleles to reach locally high frequencies or for novel alleles to be found only in individuals of mixed ancestry is well documented [54]. In these cases, the coupling of two otherwise rare alleles in a single diploid genotype may account for a non-negligible proportion of the total dataset. For example, in a study of the Passerina amoena (Lazuli bunting) and P. cyanea (Indigo bunting) hybrid zone, Carling and Brumfield [52] reported that PHASE was unable to confidently resolve genotypes of as many as 21 individuals per locus. Phylogeographic studies often detect the signals of both range expansion and secondary contact $[5,6,55,56]$. Accordingly, complex organismal histories may have a compounding effect on the number of unresolved genotypes.

\section{Impacts of omitting unresolved genotypes}

Rare alleles constitute an important component of the molecular signature used to estimate several population genetic parameters, and so omitting genotypes in which rare alleles reside could affect phylogeographic analyses. However, as noted by Edwards and Bensch [27], this has not previously been assessed. We found that systematic biases do exist, and that the absolute number of unresolved genotypes omitted from a dataset is a significant predictor of the magnitude of bias. The parameters $\Theta_{W}$ and $\pi$ tend to be underestimated (Figure 6A-D), whereas Fu's $F_{S}$ and Tajima's $D$ tend to be overestimated (Figure $6 \mathrm{E}-\mathrm{H})$. Even when only 3-4 unresolved genotypes are omitted, parameter estimates can still be quite biased (Figure 6). Although $\Theta_{W}$ and $\pi$ were mostly used for descriptive purposes in the studies included in our literature survey, they are increasingly important in phylogeographic hypothesis-testing. For example, estimates of $\Theta$ 

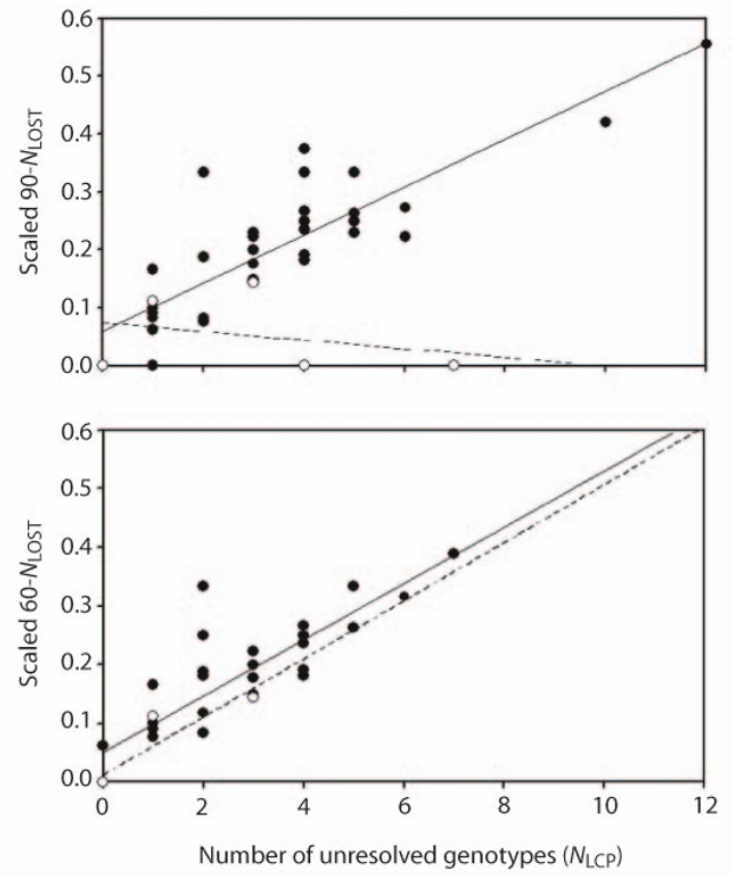

Figure 4 Relationship between the number of unresolved genotypes ( $x$-axis) and reduction in the total number of gene lineages ( $\boldsymbol{y}$-axis). Top: simulated datasets (solid circles) and empirical datasets (open circles) examined under a PHASE confidence probability threshold of 0.90. Bottom: simulated and empirical datasets examined under the 0.60 threshold. Except for the empirical data under the 0.90 threshold, all regressions were significantly positive $(P<0.0001)$.

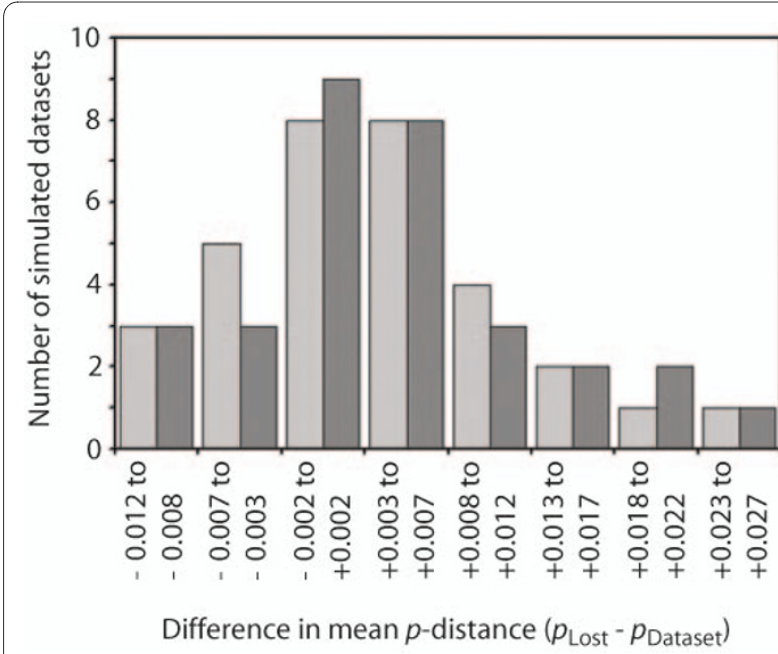

Figure 5 Frequency distribution of the difference in mean $p$-distance for only those pair-wise comparisons involving lost alleles $\left(p_{\text {LOST }}\right)$ and mean from all alleles within a dataset $\left(p_{\text {DATASET }}\right)$. Distributions for PHASE confidence probability thresholds 0.90 and 0.60 are shown in pale grey and dark grey, respectively. may be used to set effective population size $\left(N_{e}\right)$ when modelling alternative vicariance scenarios [4,21,31-33], and it is widely appreciated that fixed parameters such as $N_{e}$ can have a large impact on the outcome of such tests. The parameter $\Theta$ can also be important when ranking alternative models under an information-theoretic framework for phylogeographic inference [57]. Similarly, the combination of Tajima's $D$ and $\pi$ has been identified as particularly powerful when testing simultaneous vicariance under an approximate Bayesian computation inference framework [58].

Tests for distinguishing population growth from size constancy often use information from the frequency of distribution of DNA substitutions or haplotypes, where an excess of singletons is indicative of expansion [59]. In the absence of selection and intra-locus recombination, significantly negative values of Tajima's $D$ and Fu's $F_{S}$ are consistent with population growth. Indeed, many species have experienced rapid population expansions since the Last Glacial Maximum. In these cases, current practices of omitting unresolved genotypes should have greater impacts on analyses than those seen from simulations performed under a model of constant population size. However, even under the best-case scenario represented in simulations, our results indicate a strong upward bias in these two statistics when unresolved genotypes are omitted from a dataset (Figure 6E-H). In a highly cited paper, Ramos-Onsins and Rozas [59] reported on the superiority of Fu's $F_{S}$ to detecting population growth, and this statistic is now widely used in phylogeographic analyses. The marked overestimation of $F_{S}$ reported in the present paper could ultimately mask the signature of expansion. In a comparative phylogeographic context, understanding species' demographic histories is critical for assessing the degree to which co-distributed taxa have responded to past landscape-level events in concert $[5,6,60,61]$. Given the increasing number of studies that include re-analysis of datasets generated by other research groups $[62,63]$, it may become necessary to make a clear distinction between computationally- versus experimentally-phased nDNA sequence datasets.

Some comparative phylogeographic analyses focus on the topology and branch lengths of estimated gene trees. Here we have shown that omitting genotypes with low confidence probability scores usually leads to reductions in two components of phylogenetic diversity-the number of gene lineages and pairwise sequence divergences among them (Figure 4,5). Rare alleles can be particularly difficult to resolve (Figure 3), and so some loss of distinct gene lineages is expected. However, we also detected an unexpected bias towards loss of divergent alleles (c.f. the average $p$-distance among all alleles in the dataset). This 

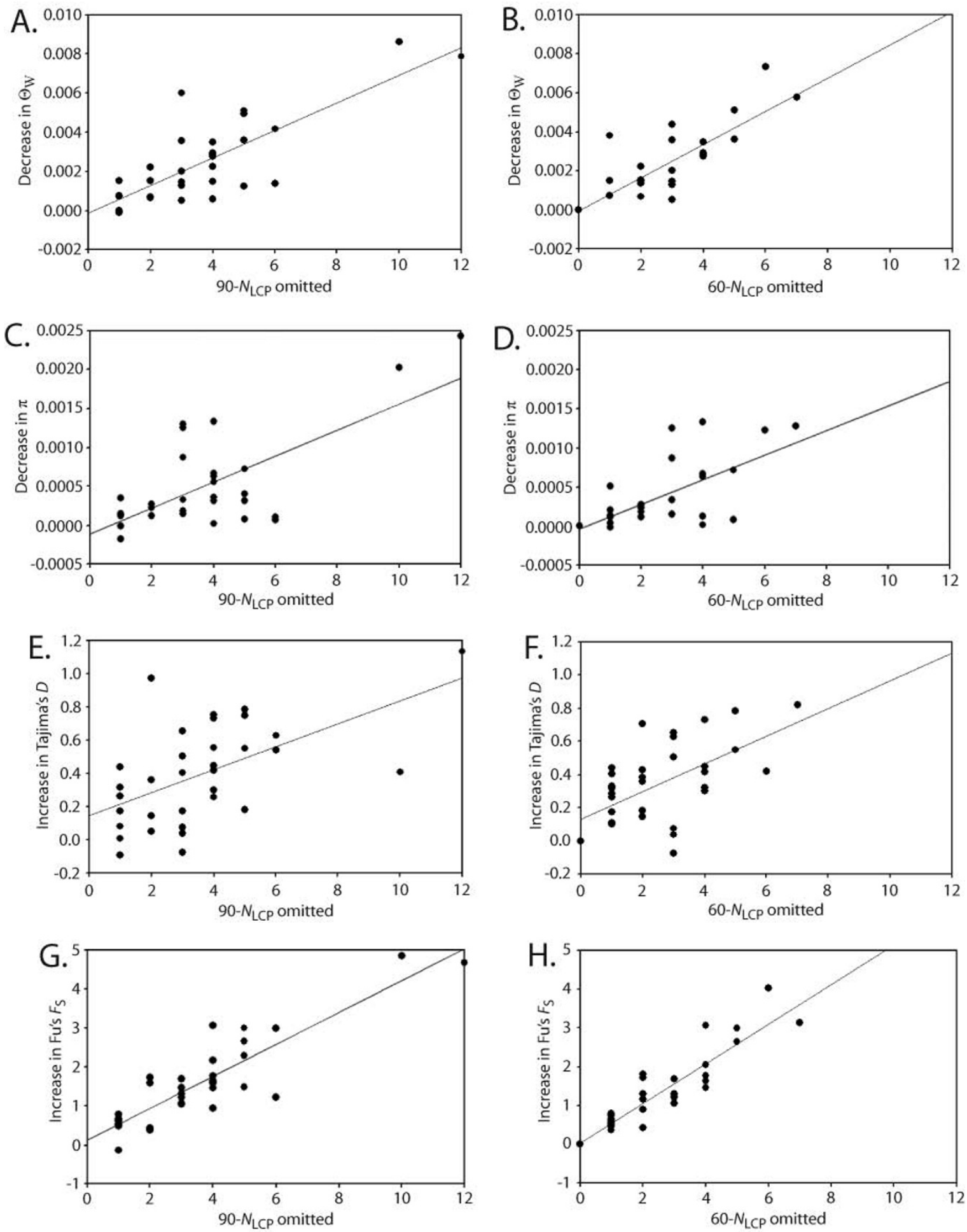

Figure 6 Relationship between the number of unresolved genotypes omitted from a dataset ( $x$-axis) and under- or over-estimation of population genetic parameters commonly used in phylogeographic analyses (y-axis). A-B, decrease in theta $\left(\Theta_{W}\right)$ under the 0.90 and 0.60 thresholds; C-D, decrease in nucleotide diversity $(\pi)$ under the 0.90 and 0.60 thresholds; E-F, increase in Tajima's D under the 0.90 and 0.60 thresholds; G-H, increase in Fu's $F_{5}$ under the 0.90 and 0.60 thresholds. 


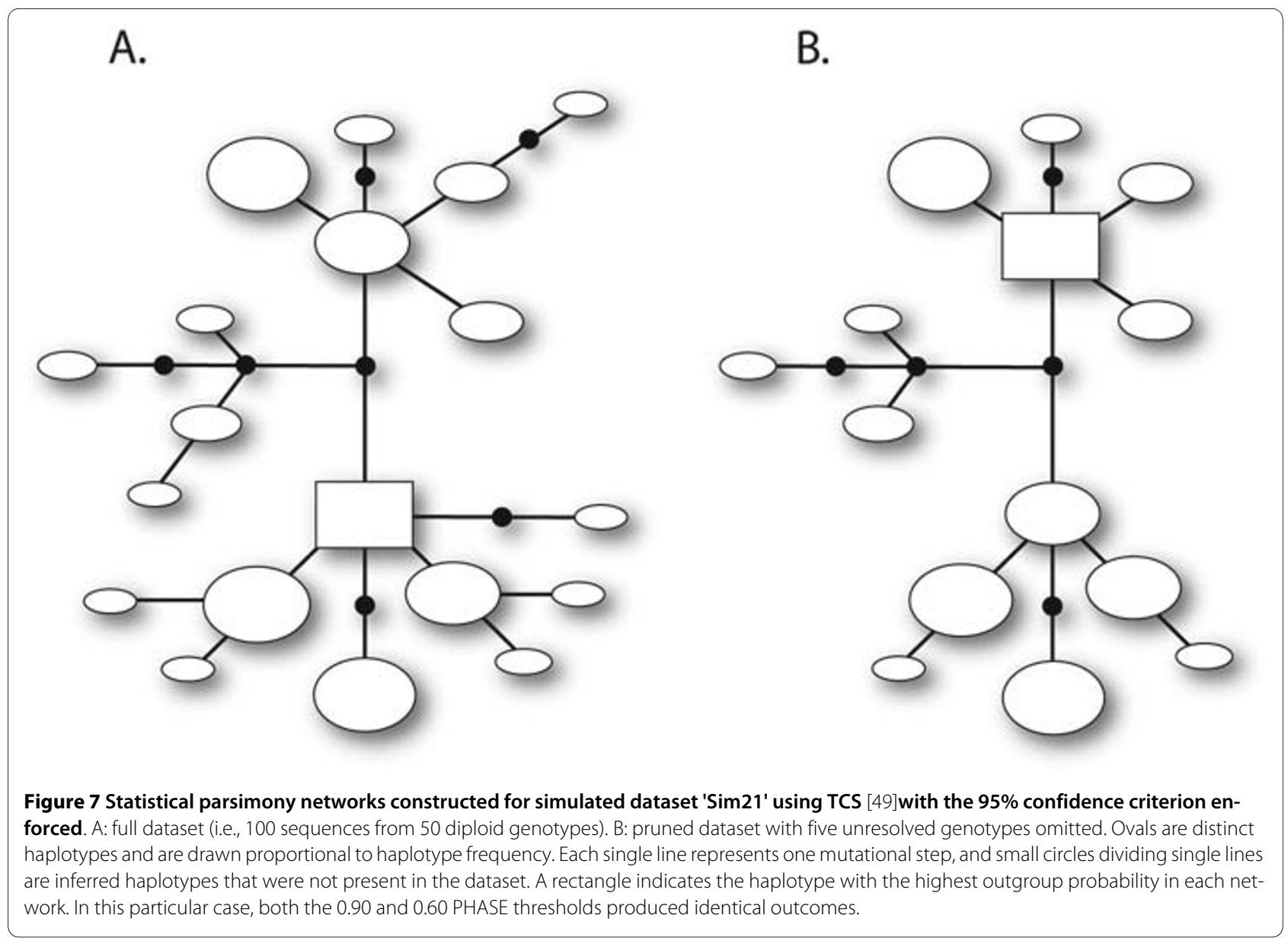

can alter estimated root probabilities in intraspecific haplotype networks (Figure 7). The implications for downstream network-based analyses (e.g., Nested Clade Phylogeographic Analysis [64]) is an area of research that demands further study, but is beyond the scope of the present paper. The systematic loss of rare alleles could also potentially impact outcomes of molecular dating methods that require removal of short branches [65], or tests of topological congruence between taxa [66]. For these reasons, some caution is warranted even when performing phylogeny-based phylogeographic analyses with computationally-phased datasets.

\section{Mitigation of observed biases and other sources of error}

We have found SSCP to be efficient for physically isolating alleles from diploid PCR products [38,41], but the utility of cloning or allele-specific PCR has also been demonstrated (Table 1). Regardless of which approach is considered most feasible, we reiterate the point made by Huang et al. [23] that the effort invested in experimental haplotype determination can be minimized by targeting genotypes that remain unresolved following computational approaches. Indeed, it may not be necessary to experimentally phase all unresolved genotypes given that biases in the four population parameter estimates examined here were always quite low when unresolved genotypes accounted for $\leq 2 \%$ of the total dataset under the 0.90 PHASE threshold (Figure 6). Notably, lowering the PHASE threshold to 0.60 often reduces the number of unresolved genotypes with little or no increase in false positives (Table 3).

The potential for some genotyping error to arise when scoring heterozygous sites from directly-sequenced diploid PCR products is well-documented. For example, base composition bias can contribute to highly asymmetric signal intensities [9], chain termination sequencing chemistry may cause certain nucleotides to produce small peaks compared to other bases at the same heterozygous position $[67,68]$, and the sequencing primers themselves can have a substantial effect on accuracy [69]. Furthermore, variable sites in close proximity to the $5^{\prime}$ or $3^{\prime}$ ends of an alignment may be difficult to score accurately [24], although it is not clear if this is a general phenomenon. Problems may also arise when one allele amplifies in PCR with low efficiency relative to another allele [70]. Taken together, if inconsistencies are seen between forward and reverse sequence chromatograms for the same diploid template, it would be prudent to experimentally validate 
these genotypes. Indeed, Bos et al.'s [53] recommendation for ground-truthing a sub-sample of the haplotypes inferred by PHASE is well justified.

\section{Conclusions}

In contrast to Harrigan et al. [24], we have not been able to escape the conclusion that a combination of experimental and computational approaches for resolving phase of segregating sites in phylogeographic applications is essential. We have shown that the current practice of omitting unresolved genotypes (i.e., those that cannot be resolved with high confidence using computational approaches implemented in PHASE) introduces systematic bias into estimates of important population genetic parameters. As expected, these biases have their most pronounced effects on summary statistics that draw on the signal embedded in the number of rare alleles (e.g., tests of neutrality or population growth). Fortunately, with targeted application of laboratory procedures that enable unambiguous phase determination via physical isolation of alleles from diploid PCR products (e.g., cloning, allele-specific PCR, SSCP), relatively little investment of time and effort is needed to overcome potential biases. This notion that the 'best' strategy involves a duality of approaches represents a recurring theme in phylogeography [71-73].

\section{Additional material}

Additional file 1 Increase over time in the use of PHASE in empirical studies relating to phylogeography, speciation or hybridization. Figure is based on the 60 articles included in our literature survey (see Table 1 in the main text). All of these studies focus on non-primate animals and used PHASE to reconstruct haplotypes from directly sequenced non-coding nuclear loci.

Additional file 2 Frequency distribution of the relationship between number of segregating sites $(S)$ and number of different alleles $\left(A_{N}\right)$ in the $\mathbf{5 0 0}$ simulated datasets from which $\mathbf{3 5}$ (solid circles) were arbitrarily selected for further analysis using PHASE. Figure shows that none of the 35 datasets are atypical (i.e., outliers), and so the results presented in the main text are free from bias relating to the dataset selection procedure.

Additional file 3 Correlation coefficients between the four measures of dataset polymorphism. In this figure, values were calculated from the pooled empirical datasets (above diagonal), and pooled simulated datasets (below diagonal). S, number of segregating sites; $A_{N}$, number of different alleles; $G_{N}$, number of different genotypes; $H_{O}$, observed heterozygosity.

Additional file $\mathbf{4}$ Supplementary references. List of 60 papers from 18 journals included in the literature survey of empirical studies that used PHASE for haplotype reconstruction (see Table 1 of the main text).

Additional file 5 Relationship between alternative measures of dataset polymorphism ( $\mathrm{x}$-axis) and the number of unresolved genotypes (y-axis). Simulated and empirical datasets are represented by solid circles and open circles, respectively. A-B, number of segregating sites $(S)$ under the 0.90 and 0.60 thresholds; $C-D$, number of different alleles $\left(A_{N}\right)$ under the 0.90 and 0.60 thresholds; $E-F$, number of different genotypes $\left(G_{N}\right)$ under the 0.90 and 0.60 thresholds; $\mathrm{G}-\mathrm{H}$, observed heterozygosity $\left(\mathrm{H}_{\mathrm{O}}\right)$ under the 0.90 and 0.60 thresholds. All regressions were significantly positive $(P<0.05)$ for simulated data, but not for the empirical data.

\section{Authors' contributions}

RCG conceived the study, performed the analyses, and drafted the paper. PS and RJD contributed ideas to the study design and interpretation of results, and revised drafts of the paper. All authors read and approved the final manuscript.

\section{Acknowledgements}

This work was supported by grants from the National Science Foundation (DEB-0543102) to RJD and John Nason, the Australian Research Council (DP0211156) to PS and Dave Rowell, and the Holsworth Wildlife Research Fund to RCG. The paper benefited from discussions during the Environmental Futures Network Phylogeography and Coalescence Workshop (Melbourne, 2007), and thoughtful comments from Saverio Vicario, Herve Philippe, and three anonymous reviewers.

\section{Author Details}

'Department of Biology, Virginia Commonwealth University, Richmond Virginia 23284, USA, ${ }^{2}$ Current Address: Department of Ecology \& Evolutionary Biology, Yale University, New Haven, Connecticut 06520, USA and ${ }^{3}$ Australian Centre for Biodiversity, School of Biological Sciences, Monash University, Clayton, Victoria 3800, Australia

Received: 18 January 2010 Accepted: 30 April 2010

Published: 30 April 2010

\section{References}

1. Brito P, Edwards SV: Multilocus phylogeography and phylogenetics using sequence-based markers. Genetica 2009, 135:439-455.

2. Friesen VL, Congdon BC, Kidd MG, Birt TP: Polymerase chain reaction primers for the amplification of five nuclear introns in vertebrates. Mol Ecol 1999, 8:2147-2149.

3. Jarman SN, Ward RD, Elliot NG: Oligonucleotide primers for PCR amplification of coelomate introns. Mar Biotechnol 2002, 4:347-355.

4. Carstens BC, Knowles LL: Shifting distributions and speciation: species divergence during rapid climate change. Mol Ecol 2007, 16:619-627.

5. Garrick RC, Rowell DM, Simmons CS, Hillis DM, Sunnucks P. Fine-scale phylogeographic congruence despite demographic incongruence in two low-mobility saproxylic springtails. Evolution 2008, 62:1103-1118.

6. Dolman G, Moritz C: A multilocus perspective on refugial isolation and divergence in rainforest skinks (Carlia). Evolution 2006, 60:573-582.

7. Lee JY, Edwards SV: Divergence across Australia's Carpentarian Barrier: statistical phylogeography of the red-backed fairy wren (Malurus melanocephalus). Evolution 2008, 62:3117-3134.

8. Avise JC: Phylogeography: retrospect and prospect. J Biogeogr 2009, 36:3-15.

9. Zhang D-X, Hewitt GM: Nuclear DNA analyses in genetic studies of populations: practice, problems and prospects. $\mathrm{Mol}$ Ecol 2003 , 12:563-584

10. Stephens M, Smith NJ, Donnelly P: A new statistical method for haplotype reconstruction from population data. Am J Hum Genet 2001, 68:978-989

11. Stephens M, Donnelly P: A comparison of Bayesian methods for haplotype reconstruction from population genotype data. Am J Hum Genet 2003, 73:1162-1169.

12. Lin S, Cutler D, Zwick ME, Chakravarti A: Haplotype inference in random population samples. Am J Hum Genet 2002, 71:1129-1137.

13. Niu T, Qin ZS, Xu X, Liu JS: Bayesian haplotype inference for multiple linked single nucleotide polymorphisms. Am J Hum Genet 2002, 70:157-169.

14. Adkins RM: Comparison of the accuracy of methods of computational haplotype inference using a large empirical dataset. BMC Genet 2004, 5:22.

15. Sabbagh A, Darlu P: Inferring haplotypes at the NAT2 locus: the computational approach. BMC Genet 2005, 6:30

16. Marchini J, Cutler D, Patterson N, Stephens M, Eskin E, Halperin E, Lin S, Qin ZS, Munro HM, Abecasis GR, Donnelly P, International HapMap Consortium: A comparison of phasing algorithms for trios and unrelated individuals. Am J Hum Genet 2006, 78:437-450

17. Bettencourt BF, Santos MR, Fialho RN, Couto AR, Peixoto MJ, Pinheiro JP, Spínola H, Mora MG, Santos C, Brehm A, Bruges-Armas J: Evaluation of 
two methods for computational HLA haplotypes inference using a real dataset. BMC Bioinformatics 2008, 9:68.

18. Lamina C, Bongardt F, Küchenhoff H, Heid IM: Haplotype reconstruction error as a classical misclassification problem: introducing sensitivity and specificity as error measures. PLOS ONE 2008, 3:e1853.

19. Tishkoff SA, Pakstis AJ, Ruano G, Kidd KK: The accuracy of statistical methods for estimation of haplotype frequencies: an example from the CD4 locus. Am J Hum Genet 2000, 67:518-522.

20. Broughton RE, Harrison RG: Nuclear gene genealogies reveal historical, demographic and selective factors associated with speciation in field crickets. Genetics 2003, 163:1389-1401.

21. Muster C, Maddison WP, Uhlmann S, Berendonk TU, Vogler AP: Arcticalpine distributions - metapopulations on a continental scale? Am Nat 2009, 173:313-326.

22. Ibrahim KM, Cooper SJB, Hewitt GM: Testing for recombination in short nuclear DNA sequence of the European meadow grasshopper, Chorthippus parallelus. Mol Ecol 2002, 11:583-590.

23. Huang Z-S, Ji Y-J, Zhang D-X: Haplotype reconstruction for scnp DNA: a consensus vote approach with extensive sequence data from populations of the migratory locust (Locusta migratoria). Mol Ecol 2008, 17:1939-1947

24. Harrigan RJ, Mazza ME, Sorenson MD: Computation vs. cloning: evaluation of two methods for haplotype determination. Mol Ecol Resour 2008, 8:1239-1248.

25. Barker JSF: Population structure and host-plant specialization in two Scaptodrosophila flower-breeding species. Heredity 2005, 94:129-138.

26. Sotka EE, Wares JP, Barth JA, Grosberg RK, Palumbi SR: Strong genetic clines and geographical variation in gene flow in the rocky intertidal barnacle Balanus glandula. Mol Ecol 2004, 13:2143-2156.

27. Edwards S, Bensch S: Looking forwards or looking backwards in avian phylogeography? A comment on Zink and Barrowclough 2008. Mol Ecol 2009, 18:2930-2933.

28. Kuhner MK, Yamato J, Felsenstein J: Maximum likelihood estimation of population growth rates based on the coalescent. Genetics 1998, 149:429-434.

29. Beerli P, Felsenstein J: Maximum likelihood estimation of a migration matrix and effective population sizes in $n$ subpopulations by using a coalescent approach. Proc Natl Acad Sci USA 2001, 98:4563-4568.

30. Hey J, Nielsen R: Multilocus methods for estimating population sizes, migration rates and divergence time, with applications to the divergence of Drosophila pseudoobscura and D. persimilis. Genetics 2004, 167:747-760.

31. Carstens BC, Degenhardt JD, Stevenson AL, Sullivan J: Accounting for coalescent stochasticity in testing phylogeographical hypotheses: modelling Pleistocene population structure in the Idaho giant salamander Dicamptodon aterrimus. Mol Ecol 2005, 14:255-265.

32. DeChaine EG, Martin AP: Using coalescent simulations to test the impact of Quaternary climate cycles on divergence in an alpine plantinsect association. Evolution 2006, 60:1004-1013.

33. Garrick RC, Nason JD, Meadows CA, Dyer RJ: Not just vicariance: phylogeography of a Sonoran Desert euphorb indicates a major role of range expansion along the Baja peninsula. Mol Ecol 2009, 18:1916-1931

34. Watterson GA: On the number of segregating sites in genetical models without recombination. Theor Popul Biol 1975, 7:256-276

35. Cornuet JM, Luikart G: Description and power analysis of two tests for detecting recent bottlenecks from allele frequency data. Genetics 1996, 144:2001-2014.

36. Fu Y-X: Statistical tests of neutrality against population growth, hitchhiking and background selection. Genetics 1997, 147:915-925.

37. Rogers AR, Harpending HC: Population growth makes waves in the distribution of pairwise genetic differences. Mol Biol Evol 1992, 9:552-569.

38. Garrick RC, Sunnucks P: Development and application of three-tiered nuclear genetic markers for basal Hexapods using single-stranded conformation polymorphism coupled with targeted DNA sequencing. BMC Genet 2006, 7:11.

39. Garrick RC, Sands CJ, Rowell DM, Tait NN, Greenslade P, Sunnucks P: Phylogeography recapitulates topography: very fine-scale local endemism of a saproxylic 'giant' springtail at Tallaganda in the Great Dividing Range of south-east Australia. Mol Ecol 2004, 13:3329-3344.
40. Garrick RC, Sands CJ, Rowell DM, Hillis DM, Sunnucks P: Catchments catch all: long-term population history of a giant springtail from the southeast Australian highlands - a multigene approach. Mol Ecol 2007, 16:1865-1882

41. Sunnucks P, Wilson ACC, Beheregaray LB, Zenger K, French J, Taylor AC SSCP is not so difficult: the application and utility of single-stranded conformation polymorphism in evolutionary biology and molecular ecology. Mol Ecol 2000, 9:1699-1710

42. Maddison WP, Maddison DR: MESQUITE: a modular system for evolutionary analysis. Distributed by the authors 2008 [http:// mesquiteproject.org].

43. Rozas J, Sánchez-DelBarrio JC, Messeguer X, Rozas R: DnaSP, DNA polymorphism analyses by the coalescent and other methods. Bioinformatics 2003, 19:2496-2497.

44. Flot J-F: SeqPHASE: a web tool for interconverting PHASE input/output files and FASTA sequence alignments. Mol Ecol Resour 2010, 10:162-166.

45. Faith DP: Conservation evaluation and phylogenetic diversity. Biol Conserv 1992, 61:1-10.

46. Tamura K, Dudley J, Nei M, Kumar S: MEGA4: Molecular Evolutionary Genetics Analysis (MEGA) software version 4.0. Mol Biol Evol 2007. 24:1596-1599.

47. Nei M: Molecular Evolutionary Genetics New York: Columbia University Press; 1987

48. Tajima F: Statistical method for testing the neutral mutation hypothesis by DNA polymorphism. Genetics 1989, 123:585-595.

49. Clement M, Posada D, Crandall KA: TCS: a computer program to estimate gene genealogies. Mol Ecol 2000, 9:1657-1659.

50. Ryynänen HJ, Primmer CR: Distribution of genetic variation in the growth hormone 1 gene in Atlantic salmon (Salmo salar) populations from Europe and North America. Mol Ecol 2004, 13:3857-3869.

51. Bigg GR, Cunningham CW, Ottersen G, Pogson GH, Wadley MR, Williamson P: Ice-age survival of Atlantic cod: agreement between palaeoecology models and genetics. Proc R Soc Lond B Biol Sci 2008, 275:163-173.

52. Carling MD, Brumfield RT: Haldane's rule in an avian system: using cline theory and divergence population genetics to test for differential introgression of mitochondrial, autosomal, and sex-linked loci across the Passerina bunting hybrid zone. Evolution 2008, 62:2600-2615.

53. Bos DH, Turner SM, DeWoody JA: Haplotype inference from diploid sequence data: evaluating performance using non-neutral MHC sequences. Hereditas 2007, 144:228-234.

54. Barton NH, Hewitt GM: Analysis of hybrid zones. Annu Rev Ecol Syst 1985, 16:113-148

55. Congdon BC, Piatt JF, Martin K, Friesen VL: Mechanisms of population differentiation in marbled murrelets: historical versus contemporary processes. Evolution 2000, 54:974-986.

56. Gifford ME, Larson A: In situ genetic differentiation in a Hispaniolan lizard (Ameiva chrysolaema): A multilocus perspective. Mol Phylogenet Evol 2008, 49:277-291.

57. Carstens BC, Stoute HN, Reid NM: An information-theoretical approach to phylogeography. Mol Ecol 2009, 18:4270-4282.

58. Hickerson MJ, Dolman G, Moritz C: Comparative phylogeographic summary statistics for testing simultaneous vicariance. Mol Ecol 2006 , 15:209-223

59. Ramos-Onsins SE, Rozas J: Statistical properties of new neutrality tests against population growth. Mol Biol Evol 2002, 19:2092-2100.

60. Sunnucks P, Blacket MJ, Taylor JM, Sands CJ, Ciavaglia SA, Garrick RC Rowell DM, Pavlova A: A tale of two flatties: different responses of two terrestrial flatworms to past environmental climatic fluctuations at Tallaganda in montane southeastern Australia. Mol Ecol 2006, 15:4513-4531

61. Kearney M, Blacket MJ: The evolution of sexual and parthenogenetic Warramaba: a window onto Plio-Pleistocene diversification processes in an arid biome. Mol Ecol 2008, 17:5257-5275.

62. Chatzimanolis S, Caterino MS: Toward a better understanding of the "Transverse Range break": lineage diversification in southern California. Evolution 2007, 61:2127-2141.

63. Leaché AD, Crews SC, Hickerson MJ: Two waves of diversification in mammals and reptiles of Baja California revealed by hierarchical Bayesian analysis. Biol Lett 2007, 3:646-650.

64. Templeton AR, Routman E, Phillips CA: Separating population structure from population history: A cladistic analysis of the geographical 
distribution of mitochondrial DNA haplotypes in the tiger salamander, Ambystoma tigrinum. Genetics 1995, 140:767-782.

65. Sanderson MJ: r8s: inferring absolute rates of molecular evolution and divergence times in the absence of a molecular clock. Bioinformatics 2003, 19:301-302.

66. Sullivan J, Arellano E, Rogers DS: Comparative phylogeography of Mesoamerican highland rodents: concerted versus independent response to climatic fluctuations. Am Nat 2000, 155:755-768.

67. Zakeri H, Amparo G, Chen SM, Spurgeon S, Kwok PY: Peak height pattern in dichloro rhodamine and energy transfer dye terminator sequencing. Biotechniques 1998, 25:406-410.

68. Simsek M, Tanira MOM, Al-Baloushi KA, Al-Barwani HS, Lawatia KM, Bayoumi RA: A precaution in the detection of heterozygotes by sequencing: comparison of automated DNA sequencing and PCRrestriction fragment length polymorphism methods. Clin Chem 2001, 47:134-137.

69. Heiden IP van der, Werf M van der, Lindemans J, van Schaik RHN: Sequencing: not always the "gold standard". Clin Chem 2004, 50:248-249

70. Cheng J, Haas M: Sensitivity of detection of heterozygous point mutations in p 53 cDNAs by direct sequencing. Genome Res 1992, 1:199-201.

71. Carstens BC, Stevenson AL, Degenhardt JD, Sullivan J: Testing nested phylogenetic and phylogeographic hypotheses in the Plethodon vandykei species group. Syst Biol 2004, 53:781-792.

72. Strasburg J, Kearney M, Moritz C, Templeton A: Combining phylogeography with distribution modeling: multiple Pleistocene range expansions in a parthenogenetic gecko from the Australian arid zone. PLOS ONE 2007, 2:e760.

73. Garrick RC, Caccone A, Sunnucks P: Inference of population history by coupling exploratory and model-driven phylogeographic analyses. Int J Mol Sci 2010, 11:1190-1227.

doi: $10.1186 / 1471-2148-10-118$

Cite this article as: Garrick et al., Nuclear gene phylogeography using

PHASE: dealing with unresolved genotypes, lost alleles, and systematic bias in parameter estimation BMC Evolutionary Biology 2010, 10:118

Submit your next manuscript to BioMed Centra and take full advantage of:

- Convenient online submission

- Thorough peer review

- No space constraints or color figure charges

- Immediate publication on acceptance

- Inclusion in PubMed, CAS, Scopus and Google Scholar

- Research which is freely available for redistribution

Submit your manuscript at www.biomedcentral.com/submit
C Biomed Central 